\title{
A Review of Coronavirus Disease-2019 (COVID-19)
}

\author{
Tanu Singhal ${ }^{1}$
}

Received: 23 February 2020 / Accepted: 25 February 2020 / Published online: 13 March 2020

(C) Dr. K C Chaudhuri Foundation 2020

\begin{abstract}
There is a new public health crises threatening the world with the emergence and spread of 2019 novel coronavirus (2019-nCoV) or the severe acute respiratory syndrome coronavirus 2 (SARS-CoV-2). The virus originated in bats and was transmitted to humans through yet unknown intermediary animals in Wuhan, Hubei province, China in December 2019. There have been around 96,000 reported cases of coronavirus disease 2019 (COVID-2019) and 3300 reported deaths to date (05/03/2020). The disease is transmitted by inhalation or contact with infected droplets and the incubation period ranges from 2 to $14 \mathrm{~d}$. The symptoms are usually fever, cough, sore throat, breathlessness, fatigue, malaise among others. The disease is mild in most people; in some (usually the elderly and those with comorbidities), it may progress to pneumonia, acute respiratory distress syndrome (ARDS) and multi organ dysfunction. Many people are asymptomatic. The case fatality rate is estimated to range from 2 to $3 \%$. Diagnosis is by demonstration of the virus in respiratory secretions by special molecular tests. Common laboratory findings include normal/ low white cell counts with elevated C-reactive protein (CRP). The computerized tomographic chest scan is usually abnormal even in those with no symptoms or mild disease. Treatment is essentially supportive; role of antiviral agents is yet to be established. Prevention entails home isolation of suspected cases and those with mild illnesses and strict infection control measures at hospitals that include contact and droplet precautions. The virus spreads faster than its two ancestors the SARS-CoV and Middle East respiratory syndrome coronavirus (MERS-CoV), but has lower fatality. The global impact of this new epidemic is yet uncertain.
\end{abstract}

Keywords 2019-nCOV $\cdot$ SARS-CoV-2 $\cdot$ COVID-19 $\cdot$ Pneumonia $\cdot$ Review

\section{Introduction}

The 2019 novel coronavirus (2019-nCoV) or the severe acute respiratory syndrome corona virus 2 (SARS-CoV-2) as it is now called, is rapidly spreading from its origin in Wuhan City of Hubei Province of China to the rest of the world [1]. Till 05/ 03/2020 around 96,000 cases of coronavirus disease 2019 (COVID-19) and 3300 deaths have been reported [2]. India has reported 29 cases till date. Fortunately so far, children have been infrequently affected with no deaths. But the future course of this virus is unknown. This article gives a bird's eye view about this new virus. Since knowledge about this virus is

Tanu Singhal

tanusinghal@yahoo.com

1 Department of Pediatrics and Infectious Disease, Kokilaben Dhirubhai Ambani Hospital and Medical Research Institute, Mumbai, India rapidly evolving, readers are urged to update themselves regularly.

\section{History}

Coronaviruses are enveloped positive sense RNA viruses ranging from $60 \mathrm{~nm}$ to $140 \mathrm{~nm}$ in diameter with spike like projections on its surface giving it a crown like appearance under the electron microscope; hence the name coronavirus [3]. Four corona viruses namely HKU1, NL63, 229E and OC43 have been in circulation in humans, and generally cause mild respiratory disease.

There have been two events in the past two decades wherein crossover of animal betacorona viruses to humans has resulted in severe disease. The first such instance was in 20022003 when a new coronavirus of the $\beta$ genera and with origin in bats crossed over to humans via the intermediary host of palm civet cats in the Guangdong province of China. This 
virus, designated as severe acute respiratory syndrome coronavirus affected 8422 people mostly in China and Hong Kong and caused 916 deaths (mortality rate $11 \%$ ) before being contained [4]. Almost a decade later in 2012, the Middle East respiratory syndrome coronavirus (MERS-CoV), also of bat origin, emerged in Saudi Arabia with dromedary camels as the intermediate host and affected 2494 people and caused 858 deaths (fatality rate $34 \%$ ) [5].

\section{Origin and Spread of COVID-19 [1, 2, 6]}

In December 2019, adults in Wuhan, capital city of Hubei province and a major transportation hub of China started presenting to local hospitals with severe pneumonia of unknown cause. Many of the initial cases had a common exposure to the Huanan wholesale seafood market that also traded live animals. The surveillance system (put into place after the SARS outbreak) was activated and respiratory samples of patients were sent to reference labs for etiologic investigations. On December 31st 2019, China notified the outbreak to the World Health Organization and on 1st January the Huanan sea food market was closed. On 7th January the virus was identified as a coronavirus that had $>95 \%$ homology with the bat coronavirus and $>70 \%$ similarity with the SARS$\mathrm{CoV}$. Environmental samples from the Huanan sea food market also tested positive, signifying that the virus originated from there [7]. The number of cases started increasing exponentially, some of which did not have exposure to the live animal market, suggestive of the fact that human-to-human transmission was occurring [8]. The first fatal case was reported on 11 th Jan 2020. The massive migration of Chinese during the Chinese New Year fuelled the epidemic. Cases in other provinces of China, other countries (Thailand, Japan and South Korea in quick succession) were reported in people who were returning from Wuhan. Transmission to healthcare workers caring for patients was described on 20th Jan, 2020. By 23rd January, the 11 million population of Wuhan was placed under lock down with restrictions of entry and exit from the region. Soon this lock down was extended to other cities of Hubei province. Cases of COVID-19 in countries outside China were reported in those with no history of travel to China suggesting that local human-to-human transmission was occurring in these countries [9]. Airports in different countries including India put in screening mechanisms to detect symptomatic people returning from China and placed them in isolation and testing them for COVID-19. Soon it was apparent that the infection could be transmitted from asymptomatic people and also before onset of symptoms. Therefore, countries including India who evacuated their citizens from Wuhan through special flights or had travellers returning from China, placed all people symptomatic or otherwise in isolation for $14 \mathrm{~d}$ and tested them for the virus.
Cases continued to increase exponentially and modelling studies reported an epidemic doubling time of $1.8 \mathrm{~d}$ [10]. In fact on the 12th of February, China changed its definition of confirmed cases to include patients with negative/ pending molecular tests but with clinical, radiologic and epidemiologic features of COVID-19 leading to an increase in cases by 15,000 in a single day [6]. As of 05/03/2020 96,000 cases worldwide (80,000 in China) and 87 other countries and 1 international conveyance (696, in the cruise ship Diamond Princess parked off the coast of Japan) have been reported [2]. It is important to note that while the number of new cases has reduced in China lately, they have increased exponentially in other countries including South Korea, Italy and Iran. Of those infected, $20 \%$ are in critical condition, $25 \%$ have recovered, and 3310 (3013 in China and 297 in other countries) have died [2]. India, which had reported only 3 cases till 2/3/ 2020, has also seen a sudden spurt in cases. By 5/3/2020, 29 cases had been reported; mostly in Delhi, Jaipur and Agra in Italian tourists and their contacts. One case was reported in an Indian who traveled back from Vienna and exposed a large number of school children in a birthday party at a city hotel. Many of the contacts of these cases have been quarantined.

These numbers are possibly an underestimate of the infected and dead due to limitations of surveillance and testing. Though the SARS-CoV-2 originated from bats, the intermediary animal through which it crossed over to humans is uncertain. Pangolins and snakes are the current suspects.

\section{Epidemiology and Pathogenesis $[10,11]$}

All ages are susceptible. Infection is transmitted through large droplets generated during coughing and sneezing by symptomatic patients but can also occur from asymptomatic people and before onset of symptoms [9]. Studies have shown higher viral loads in the nasal cavity as compared to the throat with no difference in viral burden between symptomatic and asymptomatic people [12]. Patients can be infectious for as long as the symptoms last and even on clinical recovery. Some people may act as super spreaders; a UK citizen who attended a conference in Singapore infected 11 other people while staying in a resort in the French Alps and upon return to the UK [6]. These infected droplets can spread 1-2 $\mathrm{m}$ and deposit on surfaces. The virus can remain viable on surfaces for days in favourable atmospheric conditions but are destroyed in less than a minute by common disinfectants like sodium hypochlorite, hydrogen peroxide etc. [13]. Infection is acquired either by inhalation of these droplets or touching surfaces contaminated by them and then touching the nose, mouth and eyes. The virus is also present in the stool and contamination of the water supply and subsequent transmission via aerosolization/ feco oral route is also hypothesized [6]. As per current information, transplacental transmission from pregnant women to 
their fetus has not been described [14]. However, neonatal disease due to post natal transmission is described [14]. The incubation period varies from 2 to $14 \mathrm{~d}$ [median $5 \mathrm{~d}$ ]. Studies have identified angiotensin receptor $2\left(\mathrm{ACE}_{2}\right)$ as the receptor through which the virus enters the respiratory mucosa [11].

The basic case reproduction rate (BCR) is estimated to range from 2 to 6.47 in various modelling studies [11]. In comparison, the BCR of SARS was 2 and 1.3 for pandemic flu H1N1 2009 [2].

\section{Clinical Features [8, 15-18]}

The clinical features of COVID-19 are varied, ranging from asymptomatic state to acute respiratory distress syndrome and multi organ dysfunction. The common clinical features include fever (not in all), cough, sore throat, headache, fatigue, headache, myalgia and breathlessness. Conjunctivitis has also been described. Thus, they are indistinguishable from other respiratory infections. In a subset of patients, by the end of the first week the disease can progress to pneumonia, respiratory failure and death. This progression is associated with extreme rise in inflammatory cytokines including IL2, IL7, IL10, GCSF, IP10, MCP1, MIP1A, and TNF $\alpha$ [15]. The median time from onset of symptoms to dyspnea was $5 \mathrm{~d}$, hospitalization $7 \mathrm{~d}$ and acute respiratory distress syndrome (ARDS) 8 d. The need for intensive care admission was in $25-30 \%$ of affected patients in published series. Complications witnessed included acute lung injury, ARDS, shock and acute kidney injury. Recovery started in the 2 nd or 3 rd wk. The median duration of hospital stay in those who recovered was $10 \mathrm{~d}$. Adverse outcomes and death are more common in the elderly and those with underlying co-morbidities (50-75\% of fatal cases). Fatality rate in hospitalized adult patients ranged from 4 to $11 \%$. The overall case fatality rate is estimated to range between 2 and $3 \%$ [2].

Interestingly, disease in patients outside Hubei province has been reported to be milder than those from Wuhan [17]. Similarly, the severity and case fatality rate in patients outside China has been reported to be milder [6]. This may either be due to selection bias wherein the cases reporting from Wuhan included only the severe cases or due to predisposition of the Asian population to the virus due to higher expression of $\mathrm{ACE}_{2}$ receptors on the respiratory mucosa [11].

Disease in neonates, infants and children has been also reported to be significantly milder than their adult counterparts. In a series of 34 children admitted to a hospital in Shenzhen, China between January 19th and February 7th, there were 14 males and 20 females. The median age was 8 y $11 \mathrm{mo}$ and in 28 children the infection was linked to a family member and 26 children had history of travel/residence to Hubei province in China. All the patients were either asymptomatic $(9 \%)$ or had mild disease. No severe or critical cases were seen. The most common symptoms were fever $(50 \%)$ and cough $(38 \%)$. All patients recovered with symptomatic therapy and there were no deaths. One case of severe pneumonia and multiorgan dysfunction in a child has also been reported [19]. Similarly the neonatal cases that have been reported have been mild [20].

\section{Diagnosis [21]}

A suspect case is defined as one with fever, sore throat and cough who has history of travel to China or other areas of persistent local transmission or contact with patients with similar travel history or those with confirmed COVID-19 infection. However cases may be asymptomatic or even without fever. A confirmed case is a suspect case with a positive molecular test.

Specific diagnosis is by specific molecular tests on respiratory samples (throat swab/ nasopharyngeal swab/ sputum/ endotracheal aspirates and bronchoalveolar lavage). Virus may also be detected in the stool and in severe cases, the blood. It must be remembered that the multiplex PCR panels currently available do not include the COVID-19. Commercial tests are also not available at present. In a suspect case in India, the appropriate sample has to be sent to designated reference labs in India or the National Institute of Virology in Pune. As the epidemic progresses, commercial tests will become available.

Other laboratory investigations are usually non specific. The white cell count is usually normal or low. There may be lymphopenia; a lymphocyte count $<1000$ has been associated with severe disease. The platelet count is usually normal or mildly low. The CRP and ESR are generally elevated but procalcitonin levels are usually normal. A high procalcitonin level may indicate a bacterial co-infection. The ALT/AST, prothrombin time, creatinine, D-dimer, CPK and LDH may be elevated and high levels are associated with severe disease.

The chest X-ray (CXR) usually shows bilateral infiltrates but may be normal in early disease. The CT is more sensitive and specific. CT imaging generally shows infiltrates, ground glass opacities and sub segmental consolidation. It is also abnormal in asymptomatic patients/ patients with no clinical evidence of lower respiratory tract involvement. In fact, abnormal CT scans have been used to diagnose COVID-19 in suspect cases with negative molecular diagnosis; many of these patients had positive molecular tests on repeat testing [22].

\section{Differential Diagnosis [21]}

The differential diagnosis includes all types of respiratory viral infections [influenza, parainfluenza, respiratory syncytial virus (RSV), adenovirus, human metapneumovirus, non 
COVID-19 coronavirus], atypical organisms (mycoplasma, chlamydia) and bacterial infections. It is not possible to differentiate COVID-19 from these infections clinically or through routine lab tests. Therefore travel history becomes important. However, as the epidemic spreads, the travel history will become irrelevant.

\section{Treatment $[21,23]$}

Treatment is essentially supportive and symptomatic.

The first step is to ensure adequate isolation (discussed later) to prevent transmission to other contacts, patients and healthcare workers. Mild illness should be managed at home with counseling about danger signs. The usual principles are maintaining hydration and nutrition and controlling fever and cough. Routine use of antibiotics and antivirals such as oseltamivir should be avoided in confirmed cases. In hypoxic patients, provision of oxygen through nasal prongs, face mask, high flow nasal cannula (HFNC) or non-invasive ventilation is indicated. Mechanical ventilation and even extra corporeal membrane oxygen support may be needed. Renal replacement therapy may be needed in some. Antibiotics and antifungals are required if co-infections are suspected or proven. The role of corticosteroids is unproven; while current international consensus and WHO advocate against their use, Chinese guidelines do recommend short term therapy with low-to-moderate dose corticosteroids in COVID-19 ARDS [24, 25]. Detailed guidelines for critical care management for COVID-19 have been published by the WHO [26]. There is, as of now, no approved treatment for COVID-19. Antiviral drugs such as ribavirin, lopinavirritonavir have been used based on the experience with SARS and MERS. In a historical control study in patients with SARS, patients treated with lopinavir-ritonavir with ribavirin had better outcomes as compared to those given ribavirin alone [15].

In the case series of 99 hospitalized patients with COVID19 infection from Wuhan, oxygen was given to $76 \%$, noninvasive ventilation in $13 \%$, mechanical ventilation in $4 \%$, extracorporeal membrane oxygenation (ECMO) in 3\%, continuous renal replacement therapy (CRRT) in 9\%, antibiotics in $71 \%$, antifungals in $15 \%$, glucocorticoids in $19 \%$ and intravenous immunoglobulin therapy in $27 \%$ [15]. Antiviral therapy consisting of oseltamivir, ganciclovir and lopinavirritonavir was given to $75 \%$ of the patients. The duration of non-invasive ventilation was 4-22 d [median $9 \mathrm{~d}$ ] and mechanical ventilation for 3-20 d [median $17 \mathrm{~d}$ ]. In the case series of children discussed earlier, all children recovered with basic treatment and did not need intensive care [17].

There is anecdotal experience with use of remdeswir, a broad spectrum anti RNA drug developed for Ebola in management of COVID-19 [27]. More evidence is needed before these drugs are recommended. Other drugs proposed for therapy are arbidol (an antiviral drug available in Russia and China), intravenous immunoglobulin, interferons, chloroquine and plasma of patients recovered from COVID-19 [21, $28,29]$. Additionally, recommendations about using traditional Chinese herbs find place in the Chinese guidelines [21].

\section{Prevention $[21,30]$}

Since at this time there are no approved treatments for this infection, prevention is crucial. Several properties of this virus make prevention difficult namely, non-specific features of the disease, the infectivity even before onset of symptoms in the incubation period, transmission from asymptomatic people, long incubation period, tropism for mucosal surfaces such as the conjunctiva, prolonged duration of the illness and transmission even after clinical recovery.

Isolation of confirmed or suspected cases with mild illness at home is recommended. The ventilation at home should be good with sunlight to allow for destruction of virus. Patients should be asked to wear a simple surgical mask and practice cough hygiene. Caregivers should be asked to wear a surgical mask when in the same room as patient and use hand hygiene every $15-20 \mathrm{~min}$.

The greatest risk in COVID-19 is transmission to healthcare workers. In the SARS outbreak of 2002, $21 \%$ of those affected were healthcare workers [31]. Till date, almost 1500 healthcare workers in China have been infected with 6 deaths. The doctor who first warned about the virus has died too. It is important to protect healthcare workers to ensure continuity of care and to prevent transmission of infection to other patients. While COVID-19 transmits as a droplet pathogen and is placed in Category B of infectious agents (highly pathogenic H5N1 and SARS), by the China National Health Commission, infection control measures recommended are those for category A agents (cholera, plague). Patients should be placed in separate rooms or cohorted together. Negative pressure rooms are not generally needed. The rooms and surfaces and equipment should undergo regular decontamination preferably with sodium hypochlorite. Healthcare workers should be provided with fit tested N95 respirators and protective suits and goggles. Airborne transmission precautions should be taken during aerosol generating procedures such as intubation, suction and tracheostomies. All contacts including healthcare workers should be monitored for development of symptoms of COVID-19. Patients can be discharged from isolation once they are afebrile for atleast $3 \mathrm{~d}$ and have two consecutive negative molecular tests at $1 \mathrm{~d}$ sampling interval. This recommendation is different from pandemic flu where patients were asked to resume work/school once afebrile for 
$24 \mathrm{~h}$ or by day 7 of illness. Negative molecular tests were not a prerequisite for discharge.

At the community level, people should be asked to avoid crowded areas and postpone non-essential travel to places with ongoing transmission. They should be asked to practice cough hygiene by coughing in sleeve/ tissue rather than hands and practice hand hygiene frequently every $15-20 \mathrm{~min}$. Patients with respiratory symptoms should be asked to use surgical masks. The use of mask by healthy people in public places has not shown to protect against respiratory viral infections and is currently not recommended by WHO. However, in China, the public has been asked to wear masks in public and especially in crowded places and large scale gatherings are prohibited (entertainment parks etc). China is also considering introducing legislation to prohibit selling and trading of wild animals [32].

The international response has been dramatic. Initially, there were massive travel restrictions to China and people returning from China/ evacuated from China are being evaluated for clinical symptoms, isolated and tested for COVID-19 for 2 wks even if asymptomatic. However, now with rapid world wide spread of the virus these travel restrictions have extended to other countries. Whether these efforts will lead to slowing of viral spread is not known.

A candidate vaccine is under development.

\section{Practice Points from an Indian Perspective}

At the time of writing this article, the risk of coronavirus in India is extremely low. But that may change in the next few weeks. Hence the following is recommended:

- Healthcare providers should take travel history of all patients with respiratory symptoms, and any international travel in the past 2 wks as well as contact with sick people who have travelled internationally.

- They should set up a system of triage of patients with respiratory illness in the outpatient department and give them a simple surgical mask to wear. They should use surgical masks themselves while examining such patients and practice hand hygiene frequently.

- Suspected cases should be referred to government designated centres for isolation and testing (in Mumbai, at this time, it is Kasturba hospital). Commercial kits for testing are not yet available in India.

- Patients admitted with severe pneumonia and acute respiratory distress syndrome should be evaluated for travel history and placed under contact and droplet isolation. Regular decontamination of surfaces should be done. They should be tested for etiology using multiplex PCR panels if logistics permit and if no pathogen is identified, refer the samples for testing for SARS-CoV-2.

- All clinicians should keep themselves updated about recent developments including global spread of the disease.

- Non-essential international travel should be avoided at this time.

- People should stop spreading myths and false information about the disease and try to allay panic and anxiety of the public.

\section{Conclusions}

This new virus outbreak has challenged the economic, medical and public health infrastructure of China and to some extent, of other countries especially, its neighbours. Time alone will tell how the virus will impact our lives here in India. More so, future outbreaks of viruses and pathogens of zoonotic origin are likely to continue. Therefore, apart from curbing this outbreak, efforts should be made to devise comprehensive measures to prevent future outbreaks of zoonotic origin.

\section{Compliance with Ethical Standards}

Conflict of Interest None.

\section{References}

1. Wang C, Horby PW, Hayden FG, Gao GF. A novel coronavirus outbreak of global health concern. Lancet. 2020. https://doi.org/10. 1016/S0140-6736(20)30185-9.

2. Coronavirus Outbreak. Available at: https://www.worldometers. info/coronavirus/. Accessed 23 Feb 2020.

3. Richman DD, Whitley RJ, Hayden FG. Clinical Virology, 4th ed. Washington: ASM Press; 2016.

4. Chan-Yeung M, Xu RH. SARS: epidemiology. Respirology. 2003;8:S9-14.

5. Middle East Respiratory Syndrome Coronavirus. Available at: https://www.who.int/emergencies/mers-cov/en/. Accessed 16 Feb 2020 .

6. World Health Organization. Situation reports. Available at: https:// www.who.int/emergencies/diseases/novel-coronavirus-2019/ situation-reports/. Accessed 22 Feb 2020.

7. Xinhua. China's CDC detects a large number of new coronaviruses in the South China seafood market in Wuhan. Available at: https:// www.xinhuanet.com/2020-01/27/c 1125504355.htm. Accessed 20 Feb 2020.

8. Huang C, Wang Y, Li X, et al. Clinical features of patients infected with 2019 novel coronavirus in Wuhan, China. Lancet. 2020;395: 497-506.

9. Rothe C, Schunk M, Sothmann P, et al. Transmission of 2019nCoV infection from an asymptomatic contact in Germany. $\mathrm{N}$ Engl J Med. 2020. https://doi.org/10.1056/NEJMc2001468.

10. Li Q, Guan X, Wu P, et al. Early transmission dynamics in Wuhan, China, of novel coronavirus-infected pneumonia. N Engl J Med. 2020. https://doi.org/10.1056/NEJMoa2001316. 
11. Cheng ZJ, Shan J. 2019 novel coronavirus: where we are and what we know. Infection. 2020:1-9. https://doi.org/10.1007/s15010-02001401-y.

12. Zou L, Ruan F, Huang M, et al. SARS-CoV-2 viral load in upper respiratory specimens of infected patients. N Engl J Med. 2020. https://doi.org/10.1056/NEJMc2001737.

13. Kampf G, Todt D, Pfaender S, Steinmann E. Persistence of coronaviruses on inanimate surfaces and its inactivation with biocidal agents. J Hosp Infect. 2020 Feb 6. pii: S0195-6701(20)30046-3.

14. Chen $\mathrm{H}$, Guo J, Wang C, et al. Clinical characteristics and intrauterine vertical transmission potential of COVID-19 infection in nine pregnant women: a retrospective review of medical records. Lancet. 2020. https://doi.org/10.1016/S0140-6736(20)30360-3.

15. Chen N, Zhou M, Dong X, et al. Epidemiological and clinical characteristics of 99 cases of 2019 novel coronavirus pneumonia in Wuhan, China: a descriptive study. Lancet. 2020;395:507-13.

16. Wang D, Hu B, Hu C, et al. Clinical characteristics of 138 hospitalized patients with 2019 novel coronavirus-infected pneumonia in Wuhan, China. JAMA. 2020. https://doi.org/10.1001/jama.2020. 1585.

17. $\mathrm{Xu} \mathrm{XW}, \mathrm{Wu} \mathrm{XX}$, Jiang $\mathrm{XG}$, et al. Clinical findings in a group of patients infected with the 2019 novel coronavirus (SARS-Cov-2) outside of Wuhan, China: retrospective case series. BMJ. 2020;368: m606.

18. Wang XF, Yuan J, Zheng YJ, et al. Clinical and epidemiological characteristics of 34 children with 2019 novel coronavirus infection in Shenzhen. [Article in Chinese]. Zhonghua Er Ke Za Zhi. 2020;58:E008

19. Chen F, Liu ZS, Zhang FR, et al. First case of severe childhood novel coronavirus pneumonia in China. Zhonghua Er Ke Za Zhi. 2020;58:E005.

20. Zeng LK, Tao XW, Yuan WH, Wang J, Liu X, Liu ZS. First case of neonate infected with novel coronavirus pneumonia in China. Zhonghua Er Ke Za Zhi. 2020;58:E009.

21. Jin YH, Cai L, Cheng ZS, et al. A rapid advice guideline for the diagnosis and treatment of 2019 novel coronavirus [2019-nCoV] infected pneumonia [standard version]. Mil Med Res. 2020;7:4.

22. Huang P, Liu T, Huang L, et al. Use of chest CT in combination with negative RT-PCR assay for the 2019 novel coronavirus but high clinical suspicion. Radiology. 2020. https://doi.org/10.1148/radiol. 2020200330.

23. Chen Z-M, Fu J-F, Shu Q, et al. Diagnosis and treatment recommendations for pediatric respiratory infection caused by the 2019 novel coronavirus. World J Pediatr. 2020:1-7. https://doi.org/10. 1007/s12519-020-00345-5.

24. Russell CD, Millar JE, Baillie JK. Clinical evidence does not support corticosteroid treatment for 2019-nCoV lung injury. Lancet. 2020;395:473-5.

25. Zhao JP, Hu Y, Du RH, et al. Expert consensus on the use of corticosteroid in patients with 2019-nCoV pneumonia. Zhonghua Jie He He Hu Xi Za Zhi. 2020;43:E007.

26. WHO. Clinical management of severe acute respiratory infection when novel coronavirus $[\mathrm{nCoV}]$ infection is suspected. Available at: https://www.who.int/publications-detail/clinical-managementof-severe-acute-respiratory-infection-when-novelcoronavirus[ncov]-infection-is-suspected. Accessed 9 Feb 2020.

27. Holshue ML, DeBolt C, Lindquist S, et al. First case of 2019 novel coronavirus in the United States. N Engl J Med. 2020. https://doi. org/10.1056/NEJMoa2001191.

28. Zhang L, Liu Y. Potential interventions for novel coronavirus in China: a systemic review. J Med Virol. 2020. https://doi.org/10. 1002/jmv.25707.

29. Multicenter Collaboration Group of Department of Science and Technology of Guangdong Province and Health Commission of Guangdong Province for Chloroquine in the Treatment of Novel Coronavirus Pneumonia. [Expert consensus on chloroquine phosphate for the treatment of novel coronavirus pneumonia]. [Article in Chinese] Zhonghua Jie He He Hu Xi Za Zhi. 2020;43:E019.

30. World Health Organization. Coronavirus disease [COVID-19] Technical Guidance: Infection Prevention and Control. Available at: https:/www.who.int/emergencies/diseases/novel-coronavirus2019/technical-guidance/infection-prevention-and-control. Accessed 20 Feb 2020.

31. Chang D, Xu H, Rebaza A, Sharma L, Dela Cruz CS. Protecting health-care workers from subclinical coronavirus infection. Lancet Respir Med. 2020. https://doi.org/10.1016/S2213-2600(20)300667.

32. Li J, Li J, Xie X, et al. Game consumption and the 2019 novel coronavirus. Lancet Infect Dis. 2020. https://doi.org/10.1016/ S1473-3099(20)30063-3

Publisher's Note Springer Nature remains neutral with regard to jurisdictional claims in published maps and institutional affiliations. 